BMJ Open

Diabetes

Research

\& Care

\title{
Metformin in gestational diabetes: the offspring follow-up (MiG TOFU): body composition and metabolic outcomes at 7-9 years of age
}

\author{
Janet A Rowan, ${ }^{1}$ Elaine C Rush, ${ }^{2}$ Lindsay D Plank, ${ }^{3}$ Jun Lu, ${ }^{2}$ Victor Obolonkin, ${ }^{2}$ \\ Suzette Coat, ${ }^{4}$ William M Hague ${ }^{4,5}$
}

\begin{abstract}
To cite: Rowan JA, Rush EC Plank LD, et al. Metformin in gestational diabetes: the offspring follow-up (MiG TOFU): body composition and metabolic outcomes at 7-9 years of age. BMJ Open Diab Res Care 2018;6:e000456. doi:10.1136/ bmjdrc-2017-000456
\end{abstract}

Received 1 August 2017 Revised 12 March 2018 Accepted 26 March 2018

\section{Check for updates}

${ }^{1}$ Department of Obstetrics, National Women's Health at Auckland City Hospital, Auckland, New Zealand ${ }^{2}$ Faculty of Health and Environmental Sciences, Auckland University of Technology, Auckland, New Zealand

${ }^{3}$ Department of Surgery, University of Auckland, Auckland, New Zealand ${ }^{4}$ Robinson Research Institute, University of Adelaide, Adelaide, South Australia, Australia ${ }^{5}$ Department of Obstetrics, Women's and Children's Hospital, Adelaide, South Australia

Correspondence to Dr Janet A Rowan; janetrowan1@gmail.com

\section{ABSTRACT}

Objective To compare body composition and metabolic outcomes at 7-9 years in offspring of women with gestational diabetes (GDM) randomized to metformin ( \pm insulin) or insulin treatment during pregnancy.

Research design and methods Children were assessed at 7 years in Adelaide $(n=109 / 181)$ and 9 years in Auckland ( $n=99 / 396)$ by anthropometry, bioimpedance analysis (BIA), dual-energy X-ray absorptiometry (DXA), magnetic resonance imaging (MRI) $(n=92 / 99)$ and fasting bloods $(\mathrm{n}=82 / 99)$.

Results In the Adelaide subgroup, mothers were similar at enrollment. Women randomized to metformin versus insulin had higher treatment glycemia $(p=0.002)$ and more infants with birth weight $>90$ th percentile $(20.7 \%$ vs $5.9 \% ; p=0.029$ ). At 7 years, there were no differences in offspring measures. In Auckland, at enrollment, women randomized to metformin had a higher body mass index $(B M I)(p=0.08)$ but gained less weight during treatment $(p=0.07)$. Offspring birth measures were similar. At 9 years, metformin offspring were larger by measures of weight, arm and waist circumferences, waist:height $(p<0.05)$; BMI, triceps skinfold $(p=0.05)$; DXA fat mass and lean mass $(p=0.07) ; \mathrm{MRl}$ abdominal fat volume $(\mathrm{p}=0.051)$. Body fat percent was similar between treatment groups by DXA and BIA. Abdominal fat percentages (visceral adipose tissue, subcutaneous adipose tissue and liver) were similar by MRI. Fasting glucose, triglyceride, insulin, insulin resistance, glycosylated hemoglobin ( $\mathrm{HbA1c})$, cholesterol, liver transaminases, leptin and adiponectin were similar. Conclusions Metformin or insulin for GDM was associated with similar offspring total and abdominal body fat percent and metabolic measures at 7-9 years. Metformin-exposed children were larger at 9 years. Metformin may interact with fetal environmental factors to influence offspring outcomes.

\section{INTRODUCTION}

The Metformin in Gestational diabetes (MiG) trial randomized women with gestational diabetes (GDM) requiring pharmacotherapy to treatment with either metformin (plus supplemental insulin if required) or insulin. ${ }^{1}$ Pregnancy outcomes were similar between the groups, although

\section{Significance of this study}

What is already known about this subject?

- It has been shown that metformin is a safe and effective treatment for women with gestational diabetes (GDM) with respect to pregnancy outcomes.

- Two-years-old offspring of women with GDM randomized to metformin or insulin treatment during pregnancy had similar total body fat percent. Metformin offspring had greater subscapular and biceps skinfolds and larger upper arm circumferences.

What are the new findings?

- This study shows that offspring of women with GDM randomized to metformin or insulin treatment during pregnancy have similar total and abdominal body fat percent and metabolic measures at 7-9 years of age.

- The 9-years-old offspring of women randomized to metformin were larger than those whose mothers had been randomized to insulin.

How might these results change the focus of research or clinical practice?

- Longer term follow-up of offspring of women with GDM, especially those exposed to metformin, will determine how body composition and metabolic measures at 7-9 years relate to later metabolic health.

- Our data, when considered with animal data, suggest that future research could examine how metformin might interact with other intrauterine nutritional factors to influence long-term outcomes for offspring of women with GDM. 
have been performed, and recent meta-analyses have concluded that metformin is a safe alternative to insulin for pregnancy outcomes. ${ }^{2-6}$ However, metformin crosses the placenta, and a lack of long-term offspring follow-up (TOFU) data has led to caution about using metformin routinely in GDM.

The offspring of women enrolled in the MiG trial have been followed at two centers: Auckland and Adelaide. Body composition was examined in a subgroup at 2 years of age (MiG TOFU 2 years of age) ${ }^{7}$ Body fat percent measured by dual-energy X-ray absorptiometry (DXA) and bioimpedance analysis (BIA) was no different between the treatment arms, but mid-upper arm circumference was larger, and biceps and subscapular skinfolds were greater in offspring of women randomized to metformin. These findings raised a question as to whether metformin exposure in utero was associated with a different pattern of fat deposition and whether this would persist and/or be associated with differences in insulin sensitivity. The MiG TOFU 2-year data also showed similar blood pressure and neurodevelopmental scores between treatment arms. ${ }^{89}$ We have also reported that in the total group at 2 years, size related to gender and ethnicity. ${ }^{10}$ Other small studies from women with polycystic ovarian syndrome (PCOS) have reported children of mothers treated with metformin being similar in size or weighing more than a control group, and there is a report of higher fasting glucose and blood pressure in twelve 8-years-old children previously exposed to metformin in utero, compared with normal controls. ${ }^{11-14}$

Studies in rodents have reported that metformin administration to an obese/high-fat diet (HFD) pregnant dam was beneficial to subsequent adiposity and metabolism of the offspring. ${ }^{1516}$ However, when metformin was given during pregnancy to a lean dam being fed her usual chow or a genetically obese dam that reduced her food intake and gained less weight, there were subsequent gender-specific adverse effects on offspring adiposity and metabolism. ${ }^{1718}$ These data suggest that fetal nutrient supply, gender and postnatal environment are likely to be important additional factors to consider when examining effects of metformin.

The aim of the MiG TOFU 7-9 years of age follow-up study was to compare body composition and markers of insulin sensitivity in offspring of women with GDM randomized to metformin (plus supplemental insulin as required) or insulin.

\section{RESEARCH DESIGN AND METHODS}

This was a longitudinal follow-up study of the offspring of women with GDM recruited into a prospective randomized trial comparing metformin with insulin treatment (MiG trial). ${ }^{1}$ The TOFU study is registered with the Australasian Clinical Trials Registry (ACTRN12605000311651). Women randomized into the MiG trial who agreed to follow-up have been contacted on an annual basis. In two sites, Adelaide and Auckland, measurements at approximately 7 years and 9 years of age, respectively, were undertaken.

At both centers, written informed consent was obtained from the mother/guardian of the child, and all children gave verbal consent before each procedure.

Baseline demographic and lifestyle data were collected by questionnaires. Tanner stage of development was also assessed by parental questionnaire, using pictures depicting different stages of pubertal development. Assessments were performed at the local hospital or university departments where facilities were available to measure body composition. At both sites, anthropometric and bioimpedance (BIA) measures were taken, using the protocol that was used when the children were measured at 2 years of age. ${ }^{7}$ The BIA fat-free mass was calculated using an equation developed and validated in New Zealand with the same instrumentation and protocols. ${ }^{19}$

Whole-body DXA for total body fat, fat-free mass and percent body fat was performed in Auckland using a GE-Lunar iDXA (software V.15) and in Adelaide using a GE-Lunar Prodigy (software V.13.6, GE-Lunar, Madison, Wisconsin, USA) with standard imaging and positioning protocols. Abdominal fat was determined from a region of interest (ROI) automatically defined with lower boundary placed at the top of the iliac crest and height set at $20 \%$ of the distance from this limit to the base of the skull. Thigh fat was determined from an automatically defined ROI with upper boundary set at 1.5 times the height of the abdominal ROI below the abdominal lower boundary. Height of this ROI was twice that of the abdominal ROI.

In Auckland, abdominal MRI and liver magnetic resonance spectroscopy (MRS) were also performed, if consented. In Adelaide, 12 MRIs were performed at a later date, when the children were closer to 10 years of age.

For the MRI in Auckland, children were positioned on the table in a supine position, then moved into a 3 Tesla MRI system (Siemens Skyra). A sagittal localizing image was acquired from $5.0 \mathrm{~mm}$ thick sections from diaphragm to pelvis. A 3D dual gradient-echo sequence acquired water/fat images in one acquisition using a two-point Dixon technique. Images were acquired using a T1-weighted spin-echo pulse sequence with a repetition time (TR) of $6.5 \mathrm{~ms}$ and an echo time (TE) out of phase/in phase, $2.4 / 4.8 \mathrm{~ms}$, flip angle $12^{\circ}$, matrix $256 \times 128$, and 0.7 number of excitations. After the abdominal scan, the child stayed in the scanner for a further $12 \mathrm{~min}$, and MRS was performed to determine the liver fat content. A $1.5 \times 1.5 \times 1.5 \mathrm{~cm}^{3}$ voxel was selected within the right lobe of the liver using images acquired from the abdominal scan. The spectrum was recorded using the stimulated-echo acquisition mode sequence, with an echo time of $20 \mathrm{~ms}$, a TR of $3000 \mathrm{~ms}$, a mixing time of $30 \mathrm{~ms}$, and 1024 data points over $1000 \mathrm{kHz}$ spectral width with 32 averages. Water-suppressed spectrum with 128 averages was also recorded to detect weak lipid 
signals. All images were obtained under free-breathing conditions.

For image analysis, first, a coarse body mask was obtained by thresholding and morphological operations (using ImageJ, NIH, Bethesda, Maryland, USA). Next, the body mask was classified into four classes (fat, water, air, and outliers) by a multivariate Gaussian intensity model, regularized by a Markov random field. The air voxels were rejected from the mask, and the adipose tissue segmentation was refined using a new 3-class Gaussian intensity model. To separate subcutaneous adipose tissue (SAT) from visceral adipose tissue (VAT), an intra-abdominal mask was created by calculating the convex hull around the water tissue. After the intra-abdominal mask was refined, all measures (the body volume, the fat percentage, the percentage of SAT and the percentage of VAT) were calculated.

For MRS analysis, water and fat peaks in each spectrum were integrated, and the area under the curve (AUC) of water peak and lipid peaks was calculated (using jMRUI 5.2). The AUC gave an estimate of the relative amount of water and fat present in the spectroscopy voxel. The fat fraction was calculated as the AUC of lipid divided by the sum of the AUCs of lipid and water.

MRI was performed in Adelaide using a multiple-slice MRI 1.5 Tesla scan (Philips Ingenia). Children were positioned within a torso array device placed overlying the abdomen. Axial T1-weighted images were obtained through the abdomen and pelvis, using a $400 \mathrm{~cm}$ field of view, with the following imaging parameters: $6 \mathrm{~mm}$ slice thickness, repetition time of $360 \mathrm{~ms}$, time to echo $21 \mathrm{~ms}$, two excitations, $90^{\circ}$ flip angle, matrix $256 \times 224$, and bandwidth 8.33 . Images were imported into a software package for analysis.

Venous blood was collected when the child first arrived for the assessments and the fasting status clarified. Samples for HbAlc and glucose measurements were sent to the hospital laboratory for same-day processing. Remaining samples were centrifuged immediately at $4^{\circ} \mathrm{C}$ and plasma stored at $-80^{\circ} \mathrm{C}$ for later batch processing. HbA1c was measured using the boronate affinity HPLC assay on a Model CLC385 analyzer (Primus, Kansas City, Missouri). Glucose was measured by a spectrophotometric enzymatic assay (Gluco-quant Glucose, Roche Diagnostics, Mannheim, Germany). An immunoturbidimetric method (Roche Diagnostics) was used for high-sensitivity $\mathrm{C}$ reactive protein assay. Enzymatic procedures on autoanalyzers were used to measure aspartate transaminase (AST) and alanine transaminase (ALT) (Model 902, Hitachi Roche, Indianapolis, Indiana) and lipid profile (Cobas c311, Roche Diagnostics). Insulin and ferritin were measured using an electrochemiluminescence immunoassay (Cobas e411, Roche Diagnostics). Quantification of plasma leptin and adiponectin was undertaken using immunoassay kits (Milliplex, Millipore, Billerica, Massachusetts, USA) and the Luminex micro-beads array system using the manufacturer's instructions (Luminex, Austin, Texas, USA). Insulin resistance was calculated using the homeostatic model assessment (HOMA) computer model (HOMA2 calculator) based on fasting glucose and insulin. ${ }^{20}$

All assessments and data entry were carried out by researchers who were blinded to the treatment allocation of the mothers.

Data were entered on Excel spreadsheets formatted a priori with validation criteria for all data entries to reduce errors within the database, which was linked to the initial $\mathrm{MiG}$ and 2-year offspring follow-up database. Database entries were checked by an independent researcher.

In Auckland, participants and their family practitioners were informed of the child's height, weight, body mass index (BMI), fasting glucose and HbA1c results within a few weeks of the assessment.

\section{Statistical analysis}

Between-group comparisons were carried out using Student's t-test for normally distributed data and MannWhitney U test for skewed data. Fisher's exact test was used for categorical variables. The significance level was set at 5\%. Potential gender and ethnicity differences were explored by subgroup analysis when possible. Statistical analysis was carried out using SAS V.9.4 and R V.3.0.2 (R Foundation for Statistical Computing, Vienna, Austria 2013). Results are expressed as mean \pm SD or median (IQR) unless otherwise stated.

Body fat percent was a key outcome measure. A study sample of 37 children in each treatment arm had $80 \%$ power to detect a $2 \%$ difference in body fat percent.

\section{RESULTS}

In total, there were 208 children assessed (28\% of the original cohort). In Adelaide, 109 children (metformin $\mathrm{n}=58$, insulin $\mathrm{n}=51$ ) were assessed and, in Auckland, 99 (metformin $\mathrm{n}=45$, insulin $\mathrm{n}=54$ ) were assessed. This represented $60.2 \%$ of the cohort recruited in Adelaide and $25.1 \%$ in Auckland (34.0\% from National Women's Health and 2.6\% from Middlemore Hospital).

Compared with women in the total MiG cohort, the women in the Adelaide group followed in this study were more likely to be European, have tertiary education and have lower measures of glycemia at enrollment $(p<0.05)$. They were less likely to have a family history of diabetes. Women in the Auckland group were more likely to have a tertiary education and a family history of diabetes but were otherwise not significantly different from the original cohort.

Six of the 99 Auckland children (three in insulin group, three in metformin group) were in early puberty (Tanner stage 2) with the remaining children being prepubertal.

\section{Seven-year follow-up: Adelaide subgroup}

Baseline characteristics of the mothers at the time they were randomized to metformin or insulin treatment during pregnancy were similar (table 1). This was a predominantly European/Caucasian population (89.7\% and $84.3 \%$ ). 
Table 1 Maternal baseline characteristics at randomization to treatment in MiG: total cohort and subgroups whose offspring were assessed at age 7 years (Adelaide) and 9 years (Auckland)

\begin{tabular}{|c|c|c|c|c|c|c|c|}
\hline & \multirow[b]{2}{*}{$\begin{array}{l}\text { Total MiG } \\
\text { cohort } n=733\end{array}$} & \multicolumn{2}{|c|}{$\begin{array}{l}\text { Subgroup whose } \\
\text { children were seen at } \\
7 \text { years (Adelaide) } n=109\end{array}$} & $\mathbf{P}$ values & \multicolumn{2}{|c|}{$\begin{array}{l}\text { Subgroup whose children } \\
\text { were seen at } 9 \text { years } \\
\text { (Auckland) } n=99\end{array}$} & \multirow{2}{*}{$\begin{array}{l}P \text { values } \\
M \text { versus } \\
I\end{array}$} \\
\hline & & $\begin{array}{l}\text { Metformin } \\
\text { (M) } n=58\end{array}$ & $\begin{array}{l}\text { Insulin (I) } \\
\mathrm{n}=51\end{array}$ & $\begin{array}{l}\text { M } \\
\text { versus I }\end{array}$ & $M n=45$ & $\begin{array}{l}l \\
n=54\end{array}$ & \\
\hline Age (years) & $32.8 \pm 5.3$ & $33.6 \pm 5.7$ & $33.9 \pm 4.7$ & 0.81 & $34.12 \pm 5.12$ & $35.21 \pm 4.72$ & 0.28 \\
\hline \multicolumn{8}{|l|}{ BMI $\left(\mathrm{kg} / \mathrm{m}^{2}\right)$} \\
\hline $\begin{array}{l}\text { At booking (before } \\
20 \text { weeks' gestation) }\end{array}$ & $32.1 \pm 7.9$ & $31.3 \pm 7.8$ & $31.9 \pm 8.3$ & 0.72 & $31.1 \pm 8.8$ & $29.5 \pm 6.4$ & 0.32 \\
\hline At enrollment & $34.9 \pm 7.8$ & $34.2 \pm 7.1$ & $34 \pm 7.9$ & 0.93 & $35.4 \pm 11.3$ & $32.0 \pm 6.3$ & 0.08 \\
\hline $\begin{array}{l}\text { Gestational age at enrollment } \\
\text { (weeks) }\end{array}$ & $30.1 \pm 3.2$ & $31.3 \pm 2.8$ & $31.6 \pm 2$ & 0.47 & $29.9 \pm 3.6$ & $29.5 \pm 3.4$ & 0.55 \\
\hline Ethnicity (self-reported) & & & & 0.17 & & & 0.39 \\
\hline European/Caucasian & $343(46.8)$ & $52(89.7)$ & $43(84.3)$ & & $25(55.6)$ & $21(38.9)$ & \\
\hline Polynesian & $156(21.3)$ & $0(0)$ & $0(0)$ & & $6(13.3)$ & $7(13.0)$ & \\
\hline Indian & $93(12.7)$ & $0(0)$ & $4(7.8)$ & & $7(15.6)$ & $16(29.6)$ & \\
\hline $\begin{array}{l}\text { Chinese and other } \\
\text { Southeast Asian }\end{array}$ & $86(11.7)$ & $4(6.9)$ & $2(3.9)$ & & $6(13.3)$ & $7(13.0)$ & \\
\hline Other or mixed & $55(7.5)$ & $2(3.4)$ & $2(3.9)$ & & $1(2.2)$ & $3(5.6)$ & \\
\hline Tertiary education & $323(44.1)$ & $30(51.7)$ & $32(62.7)$ & 0.33 & $28(62.2)$ & $32(59.3)$ & 0.84 \\
\hline Smoking in pregnancy & $121(16.5)$ & $7(12.1)$ & $1(2.0)$ & 0.065 & $5(11.1)$ & $4(7.4)$ & 0.73 \\
\hline Chronic hypertension & $58(7.9)$ & $7(12.1)$ & $5(9.8)$ & 0.77 & $7(15.6)$ & $5(9.3)$ & 0.37 \\
\hline $\begin{array}{l}\text { Family history diabetes } \\
\text { (1st degree) }\end{array}$ & $343(46.8)$ & 17 (29.3) & $20(39.2)$ & 0.31 & $25(55.6)$ & $35(64.8)$ & 0.41 \\
\hline $\begin{array}{l}\text { Fasting plasma glucose at } \\
\text { enrollment }(\mathrm{mg} / \mathrm{dL})\end{array}$ & $94 \pm 32$ & $88 \pm 16$ & $88 \pm 13$ & 0.78 & $95 \pm 16$ & $90 \pm 11$ & 0.10 \\
\hline $\begin{array}{l}\text { Fasting plasma glucose at } \\
\text { enrollment ( } \mathrm{mmol} / \mathrm{L})\end{array}$ & $5.2 \pm 1.0$ & $4.9 \pm 0.8$ & $4.9 \pm 0.7$ & 0.78 & $5.3 \pm 0.8$ & $5.0 \pm 0.6$ & 0.10 \\
\hline $\mathrm{HbA1c}$ at enrollment (\%) & $5.8 \pm 0.7$ & $5.4 \pm 0.5$ & $5.3 \pm 0.5$ & 0.76 & $5.9 \pm 0.5$ & $5.8 \pm 0.5$ & 0.30 \\
\hline $\begin{array}{l}\mathrm{HbA} 1 \mathrm{c} \text { at enrollment (mmol/ } \\
\mathrm{mol})\end{array}$ & $40 \pm 8$ & $35.5 \pm 5$ & $34 \pm 5$ & 0.76 & $41 \pm 5$ & $40 \pm 5$ & 0.30 \\
\hline $\begin{array}{l}\text { Weight gain before enrollment } \\
(\mathrm{kg})\end{array}$ & $6.7 \pm 5.4$ & $7.5 \pm 5.4$ & $6.2 \pm 4.6$ & 0.19 & $6.9 \pm 5.6$ & $6.4 \pm 4.5$ & 0.64 \\
\hline Paternal height $(\mathrm{cm})$ & $176.6 \pm 8.8$ & $179.3 \pm 10$ & $177.6 \pm 7.8$ & 0.34 & $175.2 \pm 8.8$ & $176.3 \pm 7.6$ & 0.50 \\
\hline Paternal weight (kg) & $88.1 \pm 18.4$ & $90.0 \pm 20.9$ & $88.0 \pm 13$ & 0.56 & $88.1 \pm 20.0$ & $85.7 \pm 16.5$ & 0.53 \\
\hline Paternal BMI $\left(\mathrm{kg} / \mathrm{m}^{2}\right)$ & $28.2 \pm 5.4$ & $28.0 \pm 6.2$ & $27.9 \pm 4$ & 0.90 & $28.6 \pm 5.4$ & $27.4 \pm 4.4$ & 0.29 \\
\hline
\end{tabular}

Data are expressed as mean \pm SD or $\mathrm{n}(\%)$.

$\mathrm{BMI}$, body mass index; MiG, metformin in gestational diabetes.

Several pregnancy outcomes were different between these subgroups (table 2). Glycemia during treatment, measured by four times daily capillary glucose measures (fasting and 2 hours postprandially) was higher in the metformin arm, predominantly due to a higher fasting glucose $(\mathrm{p}=0.0005)$. Also, in the metformin group, the duration of pregnancy was shorter (38.4 weeks vs 38.8 weeks; $\mathrm{p}=0.05$ ), and there were more babies $>90$ th percentile by customized birth weight charts $(20.7 \%$ vs $5.9 \%$; $p=0.03)$.

Seventy-nine $(72 \%)$ of the 109 children seen at 7 years had also been seen at 2 years of age (table 3 ).
Measurements at that time were similar between the metformin and insulin groups.

At the 7-year assessment, (table 4) the metformin subgroup was younger than the insulin subgroup by 5 months $(7.0 \pm 1.0$ years vs $7.4 \pm 1.1$ years; $\mathrm{P}=0.02)$. There was no difference in offspring gender distribution between the two treatment arms. All measures of body composition were similar in the two groups. Adjusting for age, gender and ethnicity did not reveal any differences. 
Table 2 Subgroup of children assessed at 7-9 years: maternal pregnancy and neonatal outcome data

\begin{tabular}{|c|c|c|c|c|c|c|}
\hline & \multicolumn{2}{|c|}{$\begin{array}{l}\text { Subgroup seen at } \\
7 \text { years (Adelaide) } n=109\end{array}$} & \multirow[b]{2}{*}{$P$ values } & \multicolumn{2}{|c|}{$\begin{array}{l}\text { Subgroup seen at } \\
\text { 9years (Auckland) } n=99\end{array}$} & \multirow[b]{2}{*}{$P$ values } \\
\hline & $\begin{array}{l}\text { Metformin } \\
\mathrm{n}=58\end{array}$ & $\begin{array}{l}\text { Insulin } \\
\mathrm{n}=51\end{array}$ & & $\begin{array}{l}\text { Metformin } \\
\mathrm{n}=45\end{array}$ & $\begin{array}{l}\text { Insulin } \\
\mathrm{n}=54\end{array}$ & \\
\hline \multicolumn{7}{|l|}{ Maternal } \\
\hline $\begin{array}{l}\text { Weight gain between enrollment and } 36 \text { weeks } \\
(\mathrm{kg})\end{array}$ & $1.0 \pm 2.5$ & $0.7 \pm 2.4$ & 0.59 & $0.4 \pm 3.2$ & $1.6 \pm 2.8$ & 0.07 \\
\hline Supplementary insulin prescribed & $18(31.0)$ & - & - & $23(51.1)$ & - & - \\
\hline $\begin{array}{l}\text { Glycemic control from randomization until } \\
\text { delivery * }\end{array}$ & $\mathrm{n}=57$ & $n=51$ & & $\mathrm{n}=45$ & $\mathrm{n}=54$ & \\
\hline Mean fasting capillary glucose & & & 0.0005 & & & 0.60 \\
\hline Tertile 1 (mean $83 \pm 5 \mathrm{mg} / \mathrm{dL})$ & $16(28.1)$ & $31(60.8)$ & & $17(37.8)$ & $26(48.1)$ & \\
\hline Tertile 2 (mean $92 \pm 4 \mathrm{mg} / \mathrm{dL}$ ) & $25(43.9)$ & $17(33.3)$ & & $19(42.2)$ & $19(35.2)$ & \\
\hline Tertile 3 (mean $106 \pm 11 \mathrm{mg} / \mathrm{dL}$ ) & $16(28.1)$ & $3(5.6)$ & & $9(20)$ & $9(16.7)$ & \\
\hline Mean postprandial capillary glucose & & & 0.35 & & & 0.62 \\
\hline Tertile 1 (mean $101 \pm 4$ mg/dL) & $13(22.8)$ & $18(35.3)$ & & $24(53.3)$ & $29(53.7)$ & \\
\hline Tertile 2 (mean $112 \pm 4$ mg/dL) & $28(49.1)$ & $22(43.1)$ & & $13(28.9)$ & $19(35.2)$ & \\
\hline Tertile 3 (mean $130 \pm 13 \mathrm{mg} / \mathrm{dL}$ ) & $16(28.1)$ & $11(21.6)$ & & $8(17.8)$ & $6(11.1)$ & \\
\hline Mean glucose & & & 0.002 & & & 0.64 \\
\hline Tertile 1 (94 $\pm 4 \mathrm{mg} / \mathrm{dL})$ & $12(21.1)$ & $26(51.0)$ & & $23(51.1)$ & $31(57.4)$ & \\
\hline Tertile $2(103 \pm 2 \mathrm{mg} / \mathrm{dL})$ & $30(52.6)$ & $21(41.2)$ & & $14(31.1)$ & $17(31.5)$ & \\
\hline Tertile $3(117 \pm 11 \mathrm{mg} / \mathrm{dL})$ & $15(26.3)$ & $4(7.8)$ & & $8(17.8)$ & $6(11.1)$ & \\
\hline $\mathrm{HbA1c}$ at 36 weeks (\%) & $5.3 \pm 0.6$ & $5.4 \pm 0.4$ & 0.45 & $5.7 \pm 0.5$ & $5.6 \pm 0.4$ & 0.18 \\
\hline $\mathrm{HbA} 1 \mathrm{c}$ at 36 weeks $(\mathrm{mmol} / \mathrm{mol})$ & $34 \pm 6$ & $35.5 \pm 4$ & 0.45 & $39 \pm 6$ & $38 \pm 4$ & 0.18 \\
\hline \multicolumn{7}{|l|}{ Hypertensive complications } \\
\hline Gestational hypertension & $1(1.7)$ & $0(0)$ & 1.00 & $5(11.1)$ & $3(5.5)$ & 0.46 \\
\hline Pre-eclampsia & $3(5.1)$ & $2(3.9)$ & 1.00 & $2(4.4)$ & $0(0)$ & 0.20 \\
\hline Mode of delivery & & & 0.44 & & & 0.83 \\
\hline Vaginal delivery & $33(56.9)$ & $33(64.7)$ & & $30(66.7)$ & $34(63.0)$ & \\
\hline Caesarean delivery & $25(43.1)$ & $18(35.3)$ & & 15 (33.3) & $20(37.0)$ & \\
\hline \multicolumn{7}{|l|}{ Neonatal } \\
\hline Gestational age at birth (weeks) & $38.4 \pm 1.2$ & $38.8 \pm 1.0$ & 0.05 & $38.4 \pm 1.3$ & $38.5 \pm 1.2$ & 0.75 \\
\hline Gestation at birth $<37$ weeks & $6(10.3)$ & $2(3.9)$ & 0.28 & $5(11.1 \%)$ & $6(11.1 \%)$ & 1.00 \\
\hline Birth weight (g) & $3481 \pm 565$ & $3324 \pm 431$ & 0.10 & $3284 \pm 563$ & $3238 \pm 542$ & 0.69 \\
\hline Birth weight customized centile & $61 \pm 29$ & $50 \pm 29$ & 0.053 & $49 \pm 30$ & $45 \pm 29$ & 0.59 \\
\hline Birth weight below 10 th percentile & $5(8.6)$ & $4(7.8)$ & 1.0 & $5(11.1)$ & $6(11.1)$ & 1.00 \\
\hline Birth weight above 90th percentile & $12(20.7)$ & $3(5.9)$ & 0.029 & $5(11.1)$ & $6(11.1)$ & 1.00 \\
\hline Crown-heel length (cm) & $50 \pm 2.2$ & $49.8 \pm 2$ & 0.61 & $50.4 \pm 2.7$ & $50.0 \pm 2.7$ & 0.49 \\
\hline Crown-rump length (cm) & $34.2 \pm 1.9$ & $33.8 \pm 1.6$ & 0.30 & $32.5 \pm 3.0$ & $32.4 \pm 2.6$ & 0.80 \\
\hline Head circumference $(\mathrm{cm})$ & $35.1 \pm 1.4$ & $34.6 \pm 1.2$ & 0.056 & $34.8 \pm 1.6$ & $34.5 \pm 1.7$ & 0.39 \\
\hline Chest circumference $(\mathrm{cm})$ & $34.2 \pm 2.1$ & $34 \pm 1.7$ & 0.53 & $33.7 \pm 2.7$ & $33.4 \pm 2.5$ & 0.62 \\
\hline Abdominal circumference $(\mathrm{cm})$ & $32.6 \pm 2.4$ & $31.7 \pm 2.6$ & 0.09 & $32.8 \pm 3.2$ & $32.2 \pm 2.9$ & 0.30 \\
\hline Mid-upper arm circumference (cm) & $11.2 \pm 1.0$ & $10.8 \pm 1.0$ & 0.04 & $11.1 \pm 1.5$ & $10.9 \pm 1.4$ & 0.43 \\
\hline Triceps skinfold thickness (mm) & $4.7 \pm 0.9$ & $4.3 \pm 0.9$ & 0.03 & $4.7 \pm 0.9$ & $5.0 \pm 1.1$ & 0.28 \\
\hline Subscapular skinfold thickness (mm) & $5.1 \pm 1.2$ & $4.9 \pm 1.0$ & 0.44 & $4.9 \pm 1.2$ & $4.9 \pm 1.1$ & 0.79 \\
\hline $\begin{array}{l}\text { Ponderal Index (birth weight }(\mathrm{g}) \times 100 / \text { crown- } \\
\left.\text { heel length }(\mathrm{cm})^{3}\right)\end{array}$ & $2.8 \pm 0.2$ & $2.7 \pm 0.2$ & 0.10 & $2.6 \pm 0.3$ & $2.6 \pm 0.3$ & 0.60 \\
\hline
\end{tabular}




\begin{tabular}{|c|c|c|c|c|c|c|}
\hline & \multicolumn{2}{|c|}{$\begin{array}{l}\text { Subgroup seen at } \\
7 \text { years (Adelaide) } n=109\end{array}$} & & \multicolumn{2}{|c|}{$\begin{array}{l}\text { Subgroup seen at } \\
9 \text { years (Auckland) } n=99\end{array}$} & \multirow[b]{2}{*}{$P$ values } \\
\hline & $\begin{array}{l}\text { Metformin } \\
\mathrm{n}=58\end{array}$ & $\begin{array}{l}\text { Insulin } \\
n=51\end{array}$ & $P$ values & $\begin{array}{l}\text { Metformin } \\
\mathrm{n}=45\end{array}$ & $\begin{array}{l}\text { Insulin } \\
\mathrm{n}=54\end{array}$ & \\
\hline Infant feeding 6-8 weeks postpartum & & & 0.30 & & & 0.59 \\
\hline Breast feeding & $32(55.1)$ & $25(49.0)$ & & $25(55.6)$ & $30(56.6)$ & \\
\hline Formula feeding & $17(29.3)$ & $13(25.5)$ & & $5(11.1)$ & $10(18.9)$ & \\
\hline Both breast and formula feeding & $5(8.6)$ & $13(25.5)$ & & $14(31.1)$ & $13(24.5)$ & \\
\hline Not seen & $4(6.8)$ & 0 & & $1(2.2)$ & 1 (1.9) & \\
\hline
\end{tabular}

Data are expressed as mean \pm SD or $\mathrm{n}(\%)$.

${ }^{*}$ Glucose control was calculated using capillary glucose measures during treatment. Women measured fasting and 2-hour postprandial levels daily. Control was divided into tertiles with tertile 1 reflecting women with tightest control. ${ }^{23}$

\section{Nine-year follow-up: Auckland subgroup}

The baseline maternal characteristics of the follow-up subgroup seen in Auckland at 9 years are shown in table 1 . Overall, ethnicity was not different between the two groups $(\mathrm{p}=0.17)$, but the population was heterogeneous, with $55.6 \%$ and $38.9 \%$ women in the metformin and insulin groups, respectively, of Caucasian/European ethnicity. There was a trend to a higher maternal BMI at trial enrollment in the metformin group $(35.4 \pm 11.3 \mathrm{~kg} /$ $\mathrm{m}^{2}$ vs $32.0 \pm 6.3 \mathrm{~kg} / \mathrm{m}^{2} ; \mathrm{p}=0.08$ ).

During pregnancy, maternal glycemia was similar in the two subgroups (table 2). There was a trend to less weight gain between enrollment and 36 weeks in the metformin mothers $(0.4 \pm 3.2 \mathrm{~kg}$ vs $1.6 \pm 2.8 \mathrm{~kg} ; \mathrm{P}=0.07)$. Pregnancy outcomes and neonatal birth measurements were similar.

All of the offspring seen at 9 years had been seen at 2 years (table 3 ). At that time, the metformin offspring were larger than the insulin offspring $(p<0.05)$ on several measures, including BMI, chest, mid arm, waist and hip circumferences, waist-to-height ratio and subscapular and biceps skinfolds. Body fat percent was similar by DXA $(17.3 \% \pm 5.1 \%$ vs $16.0 \pm 3.6 \% ; \mathrm{p}=0.25)$ and BIA $(17.2 \% \pm 5 \%$ vs $17.5 \pm 4.8 \%$; $\mathrm{p}=0.8$ ).

At the 9-year assessment, the metformin offspring were still larger on several measures, including weight $(37.0 \pm 12.6 \mathrm{~kg}$ vs $32.7 \pm 7.7 \mathrm{~kg} ; \mathrm{p}=0.049)$, mid-upper arm circumference $(23.0 \pm 4.3 \mathrm{~cm}$ vs $21.2 \pm 2.9 \mathrm{~cm} ; \mathrm{p}=0.02)$, waist circumference $(69.1 \pm 12.2 \mathrm{~cm}$ vs $64.2 \pm 8.4 \mathrm{~cm}$; $\mathrm{p}=0.04)$ and waist to height ratio $(0.51 \pm 0.08$ vs $0.47 \pm 0.05$; $\mathrm{p}=0.02$ ). Of borderline significance, they had a higher BMI $(p=0.051)$ and triceps skinfolds $(p=0.05)$. DXA and BIA measures also suggested that the metformin children were larger; they had a trend to higher fat-free mass ( $p=0.07$ by DXA, $p=0.065$ by BIA) and fat mass $(p=0.07$ by DXA). There was a significant difference in the upper arm fat mass in the metformin group measured by DXA $(1568 \pm 801$ vs $1285 \pm 534 \mathrm{~g} ; \mathrm{p}=0.047)$. Body fat percent was similar by DXA $(32.0 \% \pm 8.5 \%$ vs $30.3 \% \pm 6.6 \%$; $\mathrm{p}=0.28)$ and BIA $(23.6 \% \pm 8.1 \%$ vs $22.3 \% \pm 7.9 \% ; \mathrm{p}=0.43)$. Abdominal MRI measured larger fat volumes (subcutaneous fat: $3231 \pm 2412 \mathrm{~cm}^{3}$ vs $2398 \pm 1566 \mathrm{~cm}^{3}$; $\mathrm{p}=0.059$, visceral fat: $941 \pm 629 \mathrm{~cm}^{3}$ vs $722 \pm 365 \mathrm{~cm}^{3} ; \mathrm{p}=0.051$ ), but the percentage of total abdominal fat in the metformin and insulin offspring was similar $(36.0 \% \pm 14.4 \%$ vs $32.2 \% \pm 10.9 \% ; \mathrm{p}=0.16$ ), as was percentage subcutaneous fat $(27.6 \% \pm 12.6 \%$ vs $24.4 \% \pm 9.7 \%$; $\mathrm{p}=0.18)$ and visceral fat $(8.5 \% \pm 3.1 \%$ vs $7.7 \% \pm 1.9 \% ; \mathrm{p}=0.19)$. Liver fat percent by MRS was also similar (2.5 (1.1-6.1)\% vs $1.8(1.3-2.6) \%$; $\mathrm{p}=0.10)$.

Laboratory investigations (table 4) showed no differences between the groups apart from a higher ferritin in the metformin offspring $(\mathrm{p}=0.009)$. No child in either group was iron deficient. The highest ferritin concentrations were in eight children with results between $100 \mu \mathrm{g} / \mathrm{L}$ and $223.5 \mu \mathrm{g} / \mathrm{L}$. Numbers were insufficient to determine whether the higher concentrations related to other markers of inflammation.

Adjusting for age, ethnicity and gender did not change our findings. We also adjusted for maternal baseline BMI and maternal weight gain between recruitment and 36 weeks, and this did not change our findings.

We excluded the six children who had signs of early puberty and redid the analyses. This did not change the significance of our findings in general. In table 4, the weight of the children and DXA arm fat mass were no longer different (weight: $\mathrm{p}=0.07$, DXA arm fat mass: $\mathrm{p}=0.07$ ).

\section{CONCLUSIONS}

In this follow-up study of offspring aged 7 years and 9 years of women with GDM who had been randomized to metformin or insulin treatment during pregnancy, there were no differences between treatment arms in body fat percent or metabolic measures. Overall, these data are reassuring for clinicians using metformin in women with GDM. We did not combine the Adelaide and Auckland data, as the populations were different and the pregnancy outcomes and subsequent offspring measures at each site raise some interesting points for discussion which, we believe, may be of relevance for the management of GDM and use of metformin during pregnancy. 
Table 3 Subgroup of children seen at 7-9 years: measures at age 2 years

\begin{tabular}{|c|c|c|c|c|c|c|}
\hline & \multicolumn{2}{|c|}{$\begin{array}{l}7 \text { years subgroup also seen at } \\
2 \text { years (Adelaide) } n=79\end{array}$} & \multirow[b]{2}{*}{$P$ values } & \multicolumn{2}{|c|}{$\begin{array}{l}9 \text { years subgroup also seen at } \\
2 \text { years (Auckland) } n=99\end{array}$} & \multirow[b]{2}{*}{$\mathbf{P}$ values } \\
\hline & $\begin{array}{l}\text { Metformin } \\
\mathrm{n}=40\end{array}$ & $\begin{array}{l}\text { Insulin } \\
\mathrm{n}=39\end{array}$ & & $\begin{array}{l}\text { Metformin } \\
\mathrm{n}=45\end{array}$ & $\begin{array}{l}\text { Insulin } \\
\mathrm{n}=54\end{array}$ & \\
\hline Age (years) & $2.7 \pm 0.2$ & $2.7 \pm 0.2$ & 0.90 & $2.3 \pm 0.2$ & $2.3 \pm 0.3$ & 0.29 \\
\hline Male/female (n) & $25 / 15$ & $18 / 21$ & 0.22 & $28 / 17$ & $28 / 26$ & 0.32 \\
\hline Weight (kg) & $14.9 \pm 1.6$ & $14.8 \pm 2.2$ & 0.70 & $14.0 \pm 2.3$ & $13.3 \pm 1.9$ & 0.09 \\
\hline Leg length (cm) & $38.7 \pm 2.8$ & $39.4 \pm 2.8$ & 0.40 & $37.3 \pm 3.2$ & $37.7 \pm 3.7$ & 0.60 \\
\hline Head circumference $(\mathrm{cm})$ & $50.2 \pm 1.3$ & $49.7 \pm 1.7$ & 0.17 & $49.3 \pm 1.6$ & $49.0 \pm 2.1$ & 0.34 \\
\hline Chest circumference (cm) & $52.8 \pm 2.5$ & $52.2 \pm 3.0$ & 0.40 & $52.2 \pm 3.0$ & $50.7 \pm 2.7$ & 0.01 \\
\hline $\begin{array}{l}\text { Mid-upper arm } \\
\text { circumference (cm) }\end{array}$ & $16.8 \pm 1.2$ & $16.5 \pm 1.4$ & 0.25 & $17.5 \pm 1.6$ & $16.3 \pm 1.3$ & 0.0001 \\
\hline Waist:height ratio & $0.53 \pm 0.03$ & $0.53 \pm 0.02$ & 0.24 & $0.56 \pm 0.04$ & $0.54 \pm 0.04$ & 0.01 \\
\hline $\begin{array}{l}\text { Triceps skinfold thickness } \\
(\mathrm{mm})\end{array}$ & $9.5 \pm 1.9$ & $9.1 \pm 2.0$ & 0.40 & $10.2 \pm 2.2$ & $9.8 \pm 2.2$ & 0.37 \\
\hline $\begin{array}{l}\text { Subscapular skinfold } \\
\text { thickness }(\mathrm{mm})\end{array}$ & $6.2 \pm 1.3$ & $5.9 \pm 1.4$ & 0.31 & $7.2 \pm 2.1$ & $6.3 \pm 1.9$ & 0.02 \\
\hline $\begin{array}{l}\text { Biceps skinfold thickness } \\
(\mathrm{mm})\end{array}$ & $5.4 \pm 1.4$ & $5.0 \pm 1.2$ & 0.25 & $6.7 \pm 1.9$ & $5.9 \pm 1.9$ & 0.046 \\
\hline DXA & $n=11$ & $n=11$ & & $n=28$ & $\mathrm{n}=37$ & \\
\hline Fat-free mass (kg) & $12.1 \pm 1.2$ & $12.0 \pm 1.5$ & 0.80 & $11.1 \pm 1.5$ & $11.0 \pm 1.5$ & 0.79 \\
\hline Total fat (g) & $2310 \pm 643$ & $2162 \pm 390$ & 0.51 & $2406 \pm 1016$ & $2145 \pm 714$ & 0.25 \\
\hline Total fat $\%$ & $16.0 \pm 3.8$ & $15.3 \pm 2.0$ & 0.58 & $17.3 \pm 5.1$ & $16.0 \pm 3.6$ & 0.25 \\
\hline $\begin{array}{l}\text { Abdominal fat: } \% \text { of total } \\
\text { fat mass }\end{array}$ & $0.5 \pm 0.1$ & $0.4 \pm 0.1$ & 0.56 & $0.5 \pm 0.1$ & $0.5 \pm 0.5$ & 0.37 \\
\hline Bioimpedance & $\mathrm{n}=28$ & $n=31$ & & $n=33$ & $n=43$ & \\
\hline Fat-free mass $(\mathrm{kg})$ & $12.9 \pm 1.4$ & $12.7 \pm 1.8$ & 0.56 & $11.2 \pm 1.6$ & $11.0 \pm 1.5$ & 0.67 \\
\hline Total fat $\%$ & $12.8 \pm 5.5$ & $13.6 \pm 4.5$ & 0.57 & $17.2 \pm 5.0$ & $17.5 \pm 4.8$ & 0.80 \\
\hline
\end{tabular}

Data are expressed as mean \pm SD or $n(\%)$.

DXA, dual-energy $\mathrm{X}$-ray absorptiometry.

The Adelaide women had similar baseline characteristics at the time they were randomized to metformin or insulin treatment during pregnancy. They had a follow-up rate of $60.2 \%$, and the children at 2 years and 7 years of age were similar. In this subgroup (but not in the total trial population), the women treated with metformin had higher glycemia during treatment than women randomized to insulin. This was associated with the metformin children being larger at birth, which fits with other data showing that maternal glycemia relates to birth weight. ${ }^{21-23}$ Others have reported that GDM infants $>90$ th centile are more likely to develop obesity and metabolic syndrome as they grow, ${ }^{24-26}$ but we did not see this in our population. The reason for this is not clear, but animal data may provide a potential explanation, although it must be kept in mind that, in the mouse model, the early postnatal period rather than late pregnancy relates more to the third trimester in human pregnancy. In one HFD mouse model, administering metformin or placebo orally to a pregnant dam did not influence birth measures. However, metformin-exposed offspring, when fed a HFD postnatally, had less weight gain and less glucose intolerance than the placebo-exposed offspring. ${ }^{15}$ This suggests a protective effect of metformin on the offspring. It may be 
Table 4 Subgroup of children seen at 7-9 years: measures at 7-9 years

\begin{tabular}{|c|c|c|c|c|c|c|}
\hline & \multicolumn{2}{|c|}{$\begin{array}{l}\text { Subgroup seen at } 7 \text { years } \\
\text { (Adelaide) } n=109\end{array}$} & \multirow[b]{2}{*}{$P$ values } & \multicolumn{2}{|c|}{$\begin{array}{l}\text { Subgroup seen at } 9 \text { years } \\
\text { (Auckland) } n=99\end{array}$} & \multirow[b]{2}{*}{$P$ values } \\
\hline & $\begin{array}{l}\text { Metformin } \\
\mathrm{n}=58\end{array}$ & $\begin{array}{l}\text { Insulin } \\
\mathrm{n}=51\end{array}$ & & $\begin{array}{l}\text { Metformin } \\
n=45\end{array}$ & $\begin{array}{l}\text { Insulin } \\
\mathrm{n}=54\end{array}$ & \\
\hline Age (years) & $7.0 \pm 1.0$ & $7.4 \pm 1.1$ & 0.02 & $8.9 \pm 0.5$ & $8.9 \pm 0.4$ & 0.23 \\
\hline Male/female (n) & $35 / 23$ & $23 / 28$ & 0.16 & $28 / 17$ & $28 / 26$ & 0.32 \\
\hline Weight (kg) & $26.9 \pm 5.2$ & $26.3 \pm 4.9$ & 0.59 & $37.0 \pm 12.6$ & $32.7 \pm 7.7$ & 0.049 \\
\hline BMI $\left(\mathrm{kg} / \mathrm{m}^{2}\right)$ & $17.2 \pm 2.5$ & $16.9 \pm 2.5$ & 0.48 & $19.3 \pm 4.6$ & $17.7 \pm 3.0$ & 0.051 \\
\hline Leg length (cm) & $55.8 \pm 7.7$ & $57.5 \pm 3.1$ & 0.13 & $63.6 \pm 4.2$ & $63.9 \pm 4.1$ & 0.70 \\
\hline Head circumference (cm) & $52.2 \pm 1.2$ & $51.9 \pm 1.5$ & 0.24 & $53.6 \pm 2.2$ & $53.1 \pm 1.8$ & 0.23 \\
\hline Chest circumference (cm) & $63.5 \pm 6.0$ & $63.1 \pm 5.0$ & 0.66 & $70.4 \pm 10.2$ & $67.7 \pm 8.0$ & 0.16 \\
\hline Waist:height ratio & $0.48 \pm 0.05$ & $0.48 \pm 0.04$ & 0.54 & $0.51 \pm 0.08$ & $0.47 \pm 0.05$ & 0.02 \\
\hline $\begin{array}{l}\text { Triceps skinfold thickness } \\
(\mathrm{mm})\end{array}$ & $11.4 \pm 4.3$ & $11.4 \pm 4.0$ & 0.997 & $19.5 \pm 9.0$ & $16.2 \pm 6.7$ & 0.05 \\
\hline $\begin{array}{l}\text { Subscapular skinfold } \\
\text { thickness }(\mathrm{mm})\end{array}$ & $8.0 \pm 5.6$ & $7.5 \pm 5.3$ & 0.65 & $13.1 \pm 9.6$ & $10.5 \pm 6.8$ & 0.14 \\
\hline $\begin{array}{l}\text { Biceps skinfold thickness } \\
(\mathrm{mm})\end{array}$ & $6.9 \pm 3.8$ & $6.7 \pm 2.8$ & 0.72 & $13.9 \pm 7.5$ & $11.8 \pm 5.9$ & 0.14 \\
\hline DXA & $\mathrm{n}=32$ & $\mathrm{n}=29$ & & $\mathrm{n}=45$ & $\mathrm{n}=53$ & \\
\hline Fat-free mass (g) & $19702 \pm 2564$ & $19271 \pm 2532$ & 0.51 & $24385 \pm 5894$ & $22511 \pm 3689$ & 0.07 \\
\hline Total fat $\%$ & $26.8 \pm 7.6$ & $28.5 \pm 6.8$ & 0.37 & $32.0 \pm 8.5$ & $30.3 \pm 6.6$ & 0.28 \\
\hline $\begin{array}{l}\text { Abdominal fat } \% \text { of } \\
\text { abdominal mass }\end{array}$ & $21.3 \pm 11.8$ & $22.4 \pm 10.5$ & 0.71 & $29.7 \pm 14.4$ & $26.6 \pm 10.5$ & 0.24 \\
\hline Bioimpedance & $\mathrm{n}=56$ & $\mathrm{n}=51$ & & & & \\
\hline Fat-free mass $(\mathrm{kg})$ & $21.5 \pm 2.8$ & $20.7 \pm 3.0$ & 0.34 & $27.7 \pm 7.7$ & $25.1 \pm 5.2$ & 0.065 \\
\hline Total fat $\%$ & $18.8 \pm 7.9$ & $20.8 \pm 5.4$ & 0.13 & $23.6 \pm 8.1$ & $22.3 \pm 8.9$ & 0.43 \\
\hline MRI - abdomen & $\begin{array}{l}\mathrm{n}=7 \\
\text { Age: } 10.0 \pm 0.14 \\
\text { years }\end{array}$ & $\begin{array}{l}\mathrm{n}=5 \\
\text { Age: } 10.0 \pm 0.08 \\
\text { years }\end{array}$ & & $\mathrm{n}=42$ & $\mathrm{n}=50$ & \\
\hline $\begin{array}{l}\text { Abdominal fat volume } \\
\left(\mathrm{cm}^{3}\right)\end{array}$ & $2720 \pm 1786$ & $1843 \pm 724$ & 0.27 & $4172 \pm 2964$ & $3120 \pm 1898$ & 0.051 \\
\hline $\begin{array}{l}\text { Abdominal fat \% of } \\
\text { abdominal volume }\end{array}$ & $27.6 \pm 11.2$ & $23.5 \pm 9.5$ & 0.50 & $36.0 \pm 14.4$ & $32.2 \pm 10.9$ & 0.16 \\
\hline $\begin{array}{l}\text { Abdominal subcutaneous } \\
\text { fat volume }\left(\mathrm{cm}^{3}\right)\end{array}$ & $1807 \pm 1468$ & $1092 \pm 618$ & 0.28 & $3231 \pm 2412$ & $2398 \pm 1566$ & 0.059 \\
\hline $\begin{array}{l}\text { Abdominal subcutaneous } \\
\text { fat } \%\end{array}$ & $17.5 \pm 9.6$ & $14.1 \pm 8.6$ & 0.54 & $27.6 \pm 12.3$ & $24.4 \pm 9.7$ & 0.18 \\
\hline $\begin{array}{l}\text { Abdominal visceral fat } \\
\text { volume }\left(\mathrm{cm}^{3}\right)\end{array}$ & $913 \pm 610$ & $752 \pm 221$ & 0.54 & $941 \pm 629$ & $722 \pm 365$ & 0.051 \\
\hline Abdominal visceral fat \% & $10.1 \pm 4.8$ & $9.3 \pm 1.2$ & 0.69 & $8.5 \pm 3.1$ & $7.7 \pm 1.9$ & 0.19 \\
\hline
\end{tabular}




\begin{tabular}{|c|c|c|c|c|c|c|}
\hline & \multicolumn{2}{|c|}{$\begin{array}{l}\text { Subgroup seen at } 7 \text { years } \\
\text { (Adelaide) } n=109\end{array}$} & \multirow[b]{2}{*}{$P$ values } & \multicolumn{2}{|c|}{$\begin{array}{l}\text { Subgroup seen at } 9 \text { years } \\
\text { (Auckland) } n=99\end{array}$} & \multirow[b]{2}{*}{ P values } \\
\hline & $\begin{array}{l}\text { Metformin } \\
n=58\end{array}$ & $\begin{array}{l}\text { Insulin } \\
n=51\end{array}$ & & $\begin{array}{l}\text { Metformin } \\
\mathrm{n}=45\end{array}$ & $\begin{array}{l}\text { Insulin } \\
\mathrm{n}=54\end{array}$ & \\
\hline VAT:SAT & $0.74 \pm 0.41$ & $0.88 \pm 0.48$ & 0.60 & $0.35 \pm 0.15$ & $0.37 \pm 0.18$ & 0.57 \\
\hline Liver fat\% (MRS) & - & - & & $2.5(1.1-6.1)$ & $1.8(1.3-2.6)$ & 0.10 \\
\hline Venous blood & $\mathrm{n}=43$ & $\mathrm{n}=42$ & & $\begin{array}{l}n=44 \\
\text { ( } n=40 \text { for fasting } \\
\text { results) }\end{array}$ & $\begin{array}{l}n=50 \\
\text { ( } n=42 \text { for fasting } \\
\text { results) }\end{array}$ & \\
\hline $\begin{array}{l}\text { Fasting plasma glucose } \\
(\mathrm{mg} / \mathrm{dL})\end{array}$ & $85 \pm 7$ & $86 \pm 7$ & 0.14 & $85 \pm 7.0$ & $87 \pm 5.7$ & 0.10 \\
\hline $\begin{array}{l}\text { Fasting plasma glucose } \\
\text { (mmol/L) }\end{array}$ & $4.7 \pm 0.4$ & $4.8 \pm 0.4$ & 0.14 & $4.7 \pm 0.4$ & $4.8 \pm 0.3$ & 0.10 \\
\hline Ferritin $(\mu \mathrm{g} / \mathrm{L})$ & - & - & & $52(40-70)$ & $40(28-59)$ & 0.009 \\
\hline Fasting insulin (mlU/L) & - & - & & $6.5(4.6-12.4)$ & $8.6(5.9-12.2)$ & 0.24 \\
\hline Insulin resistance & - & - & & $1.0(0.6-1.6)$ & $1.1(0.8-1.6)$ & 0.31 \\
\hline $\begin{array}{l}\text { Fasting triglycerides } \\
(\mathrm{mmol} / \mathrm{L})\end{array}$ & - & - & & $0.59(0.47-0.88)$ & $0.70(0.55-0.82)$ & 0.31 \\
\hline LDL cholesterol (mmol/L) & - & - & & $2.7 \pm 0.5$ & $2.6 \pm 0.6$ & 0.81 \\
\hline HDL cholesterol (mmol/L) & - & - & & $1.6 \pm 0.4$ & $1.6 \pm 0.3$ & 0.42 \\
\hline AST (IU/L) & - & - & & $36 \pm 10$ & $33 \pm 5$ & 0.10 \\
\hline ALT (IU/L) & - & - & & $19 \pm 9$ & $17 \pm 6$ & 0.18 \\
\hline Waist circumference (cm) & $94.1 \pm 13.8$ & $93.4 \pm 18.1$ & 0.81 & $101.9 \pm 17.0$ & $100.0 \pm 15.8$ & 0.59 \\
\hline Hip circumference (cm) & $113.6 \pm 15.7$ & $116.0 \pm 17.2$ & 0.44 & $112.4 \pm 0.8$ & $111.5 \pm 14.1$ & 0.77 \\
\hline Waist:hip ratio & $0.83 \pm 0.06$ & $0.80 \pm 0.10$ & 0.12 & $0.91 \pm 0.07$ & $0.90 \pm 0.07$ & 0.43 \\
\hline \multicolumn{7}{|l|}{ Bioimpedance } \\
\hline Fat-free mass (kg) & $49.7 \pm 7.0$ & $48.9 \pm 7.4$ & 0.57 & $52.1 \pm 10.7$ & $48.9 \pm 8.9$ & 0.11 \\
\hline Total fat $(\%)$ & $38.6 \pm 8.0$ & $38.7 \pm 6.6$ & 0.96 & $37.7 \pm 6.9$ & $37.3 \pm 6.8$ & 0.82 \\
\hline \multicolumn{7}{|l|}{ Social situation } \\
\hline Single adult in household & $6(8.9)$ & 7 (14.3) & 0.58 & $5(11.1)$ & $3(5.6)$ & 0.46 \\
\hline Benefit in household & $35(62.5)$ & 31 (63.3) & 1.00 & $5(11.1)$ & $6(11.1)$ & 1.00 \\
\hline Smokers in household & $17(30.4)$ & $11(22.4)$ & 0.49 & $10(22.2)$ & $7(13.0)$ & 0.29 \\
\hline $\begin{array}{l}\text { Maternal history of } \\
\text { depression }\end{array}$ & $20(35.7)$ & $9(18.4)$ & 0.08 & 6 (13.3) & 7 (13.0) & 1.00 \\
\hline $\begin{array}{l}\text { Self-reported maternal } \\
\text { diabetes }\end{array}$ & - & - & & 19 (42.2) & $22(40.7)$ & 1.00 \\
\hline
\end{tabular}

Data are expressed as mean \pm SD, median (IQR) or $n(\%)$.

*One child in insulin subgroupin Auckland group: $\mathrm{HbA} 1 \mathrm{c}=43 \mathrm{mmol} / \mathrm{mol}$ on metformin.

ALT, alanine transaminase; AST, aspartate transaminase; BMI, body mass index; DXA, dual-energy X-ray absorptiometry; HDL, highdensity lipoprotein; LDL, low-density lipoprotein; MRS, magnetic resonance spectroscopy; SAT, subcutaneous adipose tissue; VAT, visceral adipose tissue. 
that the infants in the Adelaide group that were exposed to a higher nutrient load in utero (as measured by higher maternal glucose) were 'protected' by metformin, so that they were not more obese or glucose intolerant as they grew. This explanation also fits with several mechanisms of action of metformin in ameliorating effects of an excess fuel supply. ${ }^{27}$ Of course, there may be other explanations for our outcomes, one of which may be that our current management of GDM only influences pregnancy outcomes, but does not influence longer term outcomes, as some authors have suggested, ${ }^{28}$ and that metformin has no long-term effect on the offspring.

Auckland mothers at enrollment into the trial were not as well matched between treatment groups as the Adelaide cohort and the population was more heterogeneous. The women randomized to metformin tended to be larger than those randomized to insulin. During treatment, maternal glycemia was similar, but the metformin group had a trend to less weight gain. Pregnancy outcomes were similar, noting that the birth weight centiles were adjusted for ethnicity, maternal weight and height, gender, gestational age and parity. At 2 years of age, the metformin group was larger by a number of anthropometry measures, but body fat percent was no different. At 9 years, the metformin offspring were still larger by anthropometry measures including weight, BMI, triceps skinfold, waist and arm circumferences and waist:height ratio. Measures of fat mass/volume tended to be higher in the metformin group by DXA and MRI with similar increases in lean mass demonstrated by DXA measures. Body fat percent, as measured by BIA, DXA and MRI, and measures of glycemia and markers of insulin resistance were not different. Although adjusting for maternal BMI, maternal weight gain, ethnicity and offspring gender did not change the overall significance of our results, we note that the numbers in each subgroup were small for making adjustments, and we adjusted for factors that were not significantly different between the groups. It will be of interest to examine these factors in further analyses of the total cohort. When we previously examined all the Auckland offspring who had a DXA measurement at 2 years of age $(n=104)$, fat mass and its distribution was related to ethnicity and gender, and body fat percent was related to gender. ${ }^{10}$

If we are proposing metformin may have had a beneficial effect on the Adelaide subgroup, how does this fit with the findings of the Auckland subgroup? There are other animal models that may be of relevance to the Auckland group. In a mouse model, in which pregnant dams were lean and fed with their usual chow, metformin treatment was associated with the offspring being lighter at birth, with a similar phenotype to offspring exposed to undernutrition in utero. ${ }^{17}$ The metformin-exposed mice gained more weight postnatally, and the male offspring, when they were fed a HFD, became more adipose and more of them developed glucose intolerance than placebo-exposed offspring. ${ }^{17}$ In a different model, where genetically obese dams were fed metformin, the dams ate less and gained less weight in later pregnancy. The male offspring gained less weight postnatally, with a trend to lower fat, insulin and lipids, but the female offspring had increased weight gain, fat mass and cholesterol. ${ }^{18}$ Although the findings from these two studies are not completely consistent, they both suggest that metformin may have adverse effects if the nutrient environment for the fetus is restricted. Looking at the pregnancy outcomes in the Auckland cohort, we speculate that there was a proportion of women within the metformin group who may have reduced their calorie intake and gained inadequate weight or lost weight. We wonder if this may have led to relative 'undernutrition' for the fetus and a lower birth weight than expected from the baseline maternal characteristics. Could this in turn have led to a susceptibility to increased weight gain and other measures in some of the offspring at age 2 and 9 years of age? This could mask a beneficial effect of metformin in others. We feel these are important questions to raise, although we are unable to answer them with our data.

Putting these observations together leads us to conclude that current data from animal studies suggest important interactions between fetal nutrient supply, gender and metformin, which may influence pregnancy and long-term outcomes. ${ }^{15} 1718$ Our data are reassuring, but we need to be mindful of the animal data. In our own clinical practice, we ensure that women with GDM who are taking metformin, especially lean women, do not overly restrict their intake of carbohydrates and calories, and we emphasize the importance of maintaining ongoing appropriate weight gain. We monitor these factors at subsequent antenatal visits and recommend an increase in carbohydrate/calorie intake and add or change to insulin if there are concerns. We also stop metformin if there are signs of placental insufficiency, for example, with asymmetrical fetal growth and an abdominal circumference $<5$ th percentile on ultrasound scan (often accompanied by abnormal Dopplers and/or maternal pre-eclampsia). This practice was initially based on theoretical concerns that, as metformin ameliorates the effect of excess fuels, we did not want to move the fetal environment into one of inadequate fuel supply. In our opinion, animal data increasingly support this approach. We also believe that metformin is a useful therapeutic tool for GDM as, in most situations, the fetus is still exposed to an excess fuel supply.

It seems that, for women with GDM, benefits for pregnancy outcomes and long-term offspring outcomes may relate to a complex interplay between lifestyle factors, timing of interventions, fetal gender, glycemic control and pharmacotherapy used. Although we have some understanding about improving pregnancy outcomes, it is not clear whether this translates to improved long-term outcomes. Further studies are required to understand how longer term outcomes can also be improved.

To our knowledge, there is only one other study reporting outcomes in 8-year-old human offspring exposed to metformin in utero. ${ }^{14}$ This was a small 
follow-up study of offspring of women with PCOS, who had been randomized to metformin or placebo. The 12 children exposed to metformin had a significantly higher fasting glucose and lower low-density lipoprotein, with more males in the metformin group $(p=0.05)$. It would be of interest to know additional factors, such as maternal weight gain and diet quality during pregnancy, to see if their data are consistent with findings from animal studies.

The main strength of this study is that it is a follow-up of a randomized trial and assessors were blind to the treatment allocation of the mothers. A significant weakness is that although 208 children were seen, the overall follow-up rate was low, and the population was different from the initial MiG trial, so the results may not be applicable to the total cohort. However, the metformin and insulin groups that were compared at each site were well characterized and similar to each other at baseline, allowing valid comparisons between the groups. We note that pubertal status was determined by parental questionnaire rather than direct examination, and it is possible this could have affected results in the 9-year-old group.

The low follow-up rate, as well as limiting the ability to adjust for gender, ethnicity, and other potential factors, created additional issues. In the Adelaide subgroup, the maternal glucose control was different between the treatment groups, whereas there was no difference in the overall cohort. However, this difference allowed us to speculate on potential metformin effects, as above. In the Auckland subgroup, the baseline characteristics of the mothers were not so well matched, and the population was more heterogeneous, which made it more difficult to fully interpret some of our findings. We could have combined the Adelaide and Auckland data for larger numbers, but we believe that, for this initial report, showing interesting differences between two sites has added value. Finally, we measured many variables, which could have led to a significant finding by chance.

In conclusion, this study reports similar total and abdominal body fat percent and metabolic measures in 7-9 years old offspring of women with GDM randomized to metformin or insulin treatment during pregnancy. The 9-year-old offspring of women randomized to metformin were larger than those whose mothers had been randomized to insulin. Future studies will determine the relevance of this finding. Our data, when considered in combination with animal data, also suggest possible interactions between metformin and the intrauterine environment and raise some interesting questions for further study.

Acknowledgements The authors would like to acknowledge, in Auckland, Jewel Wen, Erin Qian and Oliver Statham for study coordination and assisting body composition measurements and Wafa Elasheg for phlebotomy and biochemical analysis, and in Adelaide, Chris Schultz for the DXA measurements, and Scott Brown for the MRI measurements. They would also like to thank icoMetrix Ltd for assisting in MRI analysis.

Contributors JAR was the principal investigator (PI) and developed the study design, contributed to the research and analysis, and wrote the manuscript. ECR was a collaborator, contributed to the study design, research in Auckland, data entry and analysis, and reviewed and edited the manuscript. LDP was a collaborator, performed the DXA measures and analysis, and oversaw the laboratory sampling, testing and analysis, and reviewed and edited the manuscript. $\mathrm{JL}$ was a collaborator, contributed to the development of the MRI measures in Auckland, analyzed the MRI and MRS data and reviewed and edited the manuscript. V0 was involved with developing the database, performed most of the analysis, and reviewed and edited the manuscript. SC was involved with setting up and performing the research in Adelaide, including data entry and analysis, and reviewed the manuscript. WMH was the PI in Adelaide, contributed to the study design, oversaw the research and data collection, and reviewed and edited the manuscript.

Funding This work was supported by the Auckland Medical Research Foundation (AMRF) in New Zealand, grant number 111013 and National Health and Medical Research Council (NHMRC) in Australia, grant number 508061.

\section{Competing interests None declared.}

Patient consent Not required.

Ethics approval The study has local ethics approval (Auckland AKX/04/08/228/ AM04, Adelaide REC1892/11/09).

Provenance and peer review Not commissioned; externally peer reviewed.

Data sharing statement There are additional unpublished data that have not yet been analyzed, as they were not specifically related to this manuscript. These include additional data around the health of the family and the child's diet and activity. There are also neurodevelopmental assessments in the 7-year-old cohort and questions about school performance and development in the 9-year-old cohort. These are available to the authors of this manuscript and their research teams at this stage, with a plan to publish further findings. The first author can be emailed with relevant queries.

Open Access This is an Open Access article distributed in accordance with the Creative Commons Attribution Non Commercial (CC BY-NC 4.0) license, which permits others to distribute, remix, adapt, build upon this work non-commercially, and license their derivative works on different terms, provided the original work is properly cited and the use is non-commercial. See: http://creativecommons.org/ licenses/by-nc/4.0/

(C) Article author(s) (or their employer(s) unless otherwise stated in the text of the article) 2018. All rights reserved. No commercial use is permitted unless otherwise expressly granted.

\section{REFERENCES}

1. Rowan JA, Hague WM, Gao W, et al. Metformin versus insulin for the treatment of gestational diabetes. N Engl J Med 2008;358:2003-15.

2. Balsells M, García-Patterson A, Solà I, et al. Glibenclamide, metformin, and insulin for the treatment of gestational diabetes: a systematic review and meta-analysis. BMJ 2015;350:h102.

3. Li G, Zhao S, Cui S, et al. Effect comparison of metformin with insulin treatment for gestational diabetes: a meta-analysis based on RCTs. Arch Gynecol Obstet 2015;292:111-20.

4. Zhao LP, Sheng XY, Zhou S, et al. Metformin versus insulin for gestational diabetes mellitus: a meta-analysis. Br J Clin Pharmacol 2015;80:1224-34.

5. Butalia S, Gutierrez L, Lodha A, et al. Short- and long-term outcomes of metformin compared with insulin alone in pregnancy: a systematic review and meta-analysis. Diabet Med 2017;34:27-36.

6. Kitwitee P, Limwattananon S, Limwattananon C, et al. Metformin for the treatment of gestational diabetes: An updated meta-analysis. Diabetes Res Clin Pract 2015;109:521-32.

7. Rowan JA, Rush EC, Obolonkin V, et al. Metformin in gestational diabetes: the offspring follow-up (MiG TOFU): body composition at 2 years of age. Diabetes Care 2011;34:2279-84.

8. Battin MR, Obolonkin V, Rush E, et al. Blood pressure measurement at two years in offspring of women randomized to a trial of metformin for GDM: follow up data from the MiG trial. BMC Pediatr 2015;15:54

9. Wouldes TA, Battin M, Coat S, et al. Neurodevelopmental outcome at 2 years in offspring of women randomised to metformin or insulin treatment for gestational diabetes. Arch Dis Child Fetal Neonatal 2016.

10. Rush EC, Obolonkin V, Battin M, et al. Body composition in offspring of New Zealand women: ethnic and gender differences at age 1-3 years in 2005-2009. Ann Hum Biol 2015;42:498-503. 
11. Glueck CJ, Goldenberg N, Pranikoff J, et al. Height, weight, and motor-social development during the first 18 months of life in 126 infants born to 109 mothers with polycystic ovary syndrome who conceived on and continued metformin through pregnancy. Hum Reprod 2004;19:1323-30.

12. ljäs $\mathrm{H}$, Vääräsmäki $M$, Saarela $T$, et al. A follow-up of a randomised study of metformin and insulin in gestational diabetes mellitus: growth and development of the children at the age of 18 months. BJOG 2015;122:994-1000.

13. Carlsen SM, Martinussen MP, Vanky E. Metformin's effect on first-year weight gain: a follow-up study. Pediatrics 2012;130:e122 2-e1226.

14. Rø TB, Ludvigsen HV, Carlsen SM, et al. Growth, body composition and metabolic profile of 8-year-old children exposed to metformin in utero. Scand J Clin Lab Invest 2012;72:570-5.

15. Salomäki $\mathrm{H}$, Heinäniemi $M$, Vähätalo $\mathrm{LH}$, et al. Prenatal metformin exposure in a maternal high fat diet mouse model alters the transcriptome and modifies the metabolic responses of the offspring. PLoS One 2014;9:e115778.

16. Tong JF, Yan X, Zhao JX, et al. Metformin mitigates the impaired development of skeletal muscle in the offspring of obese mice. Nutr Diabetes 2011;1:e7.

17. Salomäki H, Vähätalo LH, Laurila K, et al. Prenatal metformin exposure in mice programs the metabolic phenotype of the offspring during a high fat diet at adulthood. PLoS One 2013;8:e56594.

18. Salomäki-Myftari H, Vähätalo LH, Ailanen L, et al. Neuropeptide $Y$ Overexpressing Female and Male Mice Show Divergent Metabolic but Not Gut Microbial Responses to Prenatal Metformin Exposure. PLoS One 2016;11:e0163805.
19. Rush EC, Puniani K, Valencia ME, et al. Estimation of body fatness from body mass index and bioelectrical impedance: comparison of New Zealand European, Maori and Pacific Island children. Eur J Clin Nutr 2003:57:1394-401.

20. Wallace TM, Levy JC, Matthews DR. Use and abuse of HOMA modeling. Diabetes Care 2004;27:1487-95.

21. Crowther CA, Hiller JE, Moss JR, et al. Effect of treatment of gestational diabetes mellitus on pregnancy outcomes. N Engl $\mathrm{J}$ Med 2005;352:2477-86.

22. Landon MB, Spong CY, Thom E, et al. A multicenter, randomized trial of treatment for mild gestational diabetes. $N$ Engl $J$ Med 2009;361:1339-48

23. Rowan JA, Gao W, Hague WM, et al. Glycemia and its relationship to outcomes in the metformin in gestational diabetes trial. Diabetes Care 2010;33:9-16.

24. Boney CM, Verma A, Tucker R, et al. Metabolic syndrome in childhood: association with birth weight, maternal obesity, and gestational diabetes mellitus. Pediatrics 2005;115:e290-e296.

25. Franks PW, Looker HC, Kobes S, et al. Gestational glucose tolerance and risk of type 2 diabetes in young Pima Indian offspring. Diabetes 2006:55:460-5.

26. Schaefer-Graf UM, Pawliczak J, Passow D, et al. Birth weight and parental BMI predict overweight in children from mothers with gestational diabetes. Diabetes Care 2005;28:1745-50.

27. Rena G, Pearson ER, Sakamoto K. Molecular mechanism of action of metformin: old or new insights? Diabetologia 2013;56:1898-906.

28. Donovan LE, Cundy T. Does exposure to hyperglycaemia in utero increase the risk of obesity and diabetes in the offspring? Diabet Med 2016;33:695-6. 